Tropical Journal of Pharmaceutical Research October 2021; 20 (10): 2099-2107

ISSN: $1596-5996$ (print); 1596-9827 (electronic) (C) Pharmacotherapy Group, Faculty of Pharmacy, University of Benin, Benin City, 300001 Nigeria.

\title{
Luteolin prevents monoiodoacetate-induced osteoarthritis in post-menopausal rats via protection of the cartilage
}

\author{
Hao Li, Yan Gao* \\ Department of Orthopaedics, The Second Hospital of Shanxi Medical University, Taiyuan, Shanxi 030001, China \\ *For correspondence: Email: gynxgyr@126.com; Tel/Fax: 0086-0351-3232325 \\ Sent for review: 3 December 2020 \\ Revised accepted: 19 September 2021
}

\begin{abstract}
Purpose: To investigate the effectiveness of luteolin treatment in postmenopausal model of osteoarthritis (OA)

Methods: Sprague-Dawley rats were divided into five groups. Luteolin was given orally to rats at doses of 50 and $100 \mathrm{mg} / \mathrm{kg}$ for 4 months, while aceclofenac was administered at a dose of $10 \mathrm{mg} / \mathrm{kg}$. The antiinflammatory and anti-arthritic effects of luteolin and aceclofenac were determined using pawwithdrawal method. Knee joint thickness was measured using X-ray imaging. Pathological changes in bone slices were determined with immuno-histochemical evaluation. The levels of inflammatory cytokines were assessed by reverse transcription-polymerase chain reaction (RT-PCR) and western blot analysis.

Results: Oral ingestion of luteolin significantly reduced manifestations of OA and suppressed levels of serum cytokines $(p<0.05)$. Moreover, luteolin increased expression of bone marker protein and reduced the gene expression levels of matrix metalloproteinases (MMPs, $p<0.05$ ), suggesting its protective effects on chondrocytes. Luteolin significantly reduced the production of inflammatory chemokines and cytokines (IFN-y, IL-1, and IL-6). Histopathological examination showed that luteolin decreased pathological lesions in monoiodoacetate-mediated $O A$ in ovariectomized rats, indicating prevention of cartilage loss.

Conclusion: These results suggest that luteolin exerts protective effects against monoiodoacetateinduced (MIA) $O A$ in ovariectomized rats by suppressing the expressions of inflammation-related mediators (IL-1 $\beta$, Cox-2, and PGE-2). Thus, luteolin is a prospective option for the suppression of postmenopausal OA in humans.
\end{abstract}

Keywords: Luteolin, Osteoarthritis, Overiectomy, Aceclofenac

\begin{abstract}
This is an Open Access article that uses a funding model which does not charge readers or their institutions for access and distributed under the terms of the Creative Commons Attribution License (http://creativecommons.org/licenses/by/4.0) and the Budapest Open Access Initiative (http://www.budapestopenaccessinitiative.org/read), which permit unrestricted use, distribution, and
\end{abstract} reproduction in any medium, provided the original work is properly credited.

Tropical Journal of Pharmaceutical Research is indexed by Science Citation Index (SciSearch), Scopus, International Pharmaceutical Abstract, Chemical Abstracts, Embase, Index Copernicus, EBSCO, African Index Medicus, JournalSeek, Journal Citation Reports/Science Edition, Directory of Open Access Journals (DOAJ), African Journal Online, Bioline International, Open-J-Gate and Pharmacy Abstracts

\section{INTRODUCTION}

Osteoarthritis (OA) is one of the most common degenerative disorders. It manifests in clinical changes such as cartilage loss and inflammation of synovial fluid. Chondrocytes respond to OAinduced inflammation by increasing sub-chondral matrix biosynthesis and releasing antiinflammatory mediators, resulting in $\mathrm{OA}$ progression. Moreover, chondrocytes and synovial cells together produce proinflammatory cytokines which also influence apoptosis of chondrocytes [1]. Several animal models have been used to investigate the pathogenesis of OA, 
as well as treatment approaches. Ovariectomy is one of the most used strategies for induction of OA [2]. When ovariectomized rats are injected with monoiodoacetate (MIA), they turn into spontaneously advanced OA model.

The use of non-steroidal anti-inflammatory drugs (NSAIDs) and steroidal agents is associated with severe adverse effects in OA patients. Luteolin is a flavonoid present in vegetables, herbs, fruits and spices [4]. It has been shown to suppress chronic diseases, for example, OA [5]. Studies have been conducted on beneficial effect of luteolin against OA [6]. However, there are no reports on the inhibitory effect of luteolin on MIAinduced OA in ovariectomized rats.

Therefore, the present study was designed to determine the inhibitory effect of luteolin on MIAtriggered $\mathrm{OA}$ in ovariectomized rats. Knee joint was histologically examined to determine the pathological changes associated with articular cartilage erosions. In addition, the effect of luteolin on MIA-induced OA in ovariectomized rats was determined using a biochemical marker of collagen type I degradation (CTXI), in order to ascertain if the ovariectomized rats were effective post-menopausal OA model.

\section{EXPERIMENTAL}

\section{Chemicals and reagents}

Luteolin was purchased from Sigma Aldrich (Beijing, China). Monoiodoacetate (MIA), hematoxylin and eosin were brought from Sigma Aldrich (Beijing China).

\section{Animals}

Female Spraque-Dawley rats (6-week old) were obtained from Animal Research Institute, Shanxi Medical University, Shanxi, China. The rats were provided with standard rat feed and water ad libitum. The animals were acclimatized to laboratory conditions for seven days prior to the experiment. The study received approval from the Laboratory Animal Usage and Ethics Committee of the University, Shanxi (approval no. OR/SMU-20/ARI-56), and followed international guidelines for animal studies.

\section{Calculation of sample size}

The sample size was determined using power analysis $G$ power software. Standard deviation was used to determine the variability of the sample with type-1 error at $p<0.05$, and twotailed tests involving 80 percent power analysis.

\section{Establishment of ovariectomized rats}

Thirty-six-week-old virgin female SpragueDawley rats were employed. Body weight was determined at the beginning, and the animals were arbitrarily assigned to five groups of six rats each. Bilateral ovariectomy was performed on the rats under local anaesthesia with HypnormDormicum using a dorsal border incision. Rats subjected to a standard sham surgery served as control. For this purpose, a small incision was made to gain access into the abdominal cavity. The ovaries and fallopian tubes were pulled outward and removed through the incision. The incision was sealed using absorbable sutures. The ovaries were left intact without being removed, and they were considered control surgeries.

\section{Induction of osteoarthritis with MIA}

To trigger $\mathrm{OA}$, the ovariectomized rats were treated with MIA under ether anaesthesia [11]. The area below the joints of the knee was shaved and sterilized with $70 \%$ ethanol. Then, $50 \mathrm{~mL}$ of sterile phosphate buffer saline (PBS) containing monoiodoacetate (MIA, $3 \mathrm{mg}$ ) was administered intra-articularly at a volume of 50 $\mu \mathrm{L}$ into the joints of the ovariectomized rats. A simple solution of $50 \mathrm{~mL}$ PBS without MIA was administered intra-articularly into the joints of the ovariectomized rats in the control group. A period of one week was allowed for the development of OA symptoms.

\section{Study protocol}

Ovariectomized rats that developed OA after MIA injection were divided into five groups, with six rats in each group. The control rats (group 1) were subjected to sham operation in which the ovaries were exposed but not isolated. Rats in this group were administered $0.2 \mathrm{~mL}$ of PBS only, once daily, without intra-articular MIA. Group 2 comprised MIA-induced OA and osteoarthritic rats. The rats were ovariectomized and administered MIA intra-articularly. They were given only $0.2 \mathrm{~mL}$ of normal saline (vehicle) orally, once daily for 28 days, without luteolin and aceclofenac. Group 3 comprised MIA-induced $\mathrm{OA}$ and ovarictomized rats treated with luteolin (50 mg/kg, orally) once daily for 28 days, using a suspension of luteolin powder in normal saline $(0.3 \mathrm{~mL})$. Group 4 consisted of MIA-induced OA and ovariectomized rats treated orally with luteolin $(100 \mathrm{mg} / \mathrm{kg})$ for 28 days. Group 5 comprised MIA-induced OA and ovariectomized rats treated with aceclofenac $(10 \mathrm{mg} / \mathrm{kg}$, p.o.) for 28 days. 
Determination of body weight and paw withdrawal threshold

Throughout the experiment, the body weights of the rats were recorded weekly. The rats were positioned in a cage that had a wired floor mesh, and were left to acclimatize for a minimum of 5 min. Then, pain sensation was measured by assessing the paw withdrawal threshold in response to reflex stimulation from von Frey filaments, which were recorded as symptoms of OA. Every filament of von Frey was applied for 5 $\mathrm{sec}$, with an interval of $5 \mathrm{~min}$ between pain assessments.

\section{Measurement of bone degradation and thickness of knee joint swelling}

With the aid of a vernier caliper, the area of swelling in the knee joint was precisely measured post-MIA administration. The non-injected leg served as a control. Using X-ray imaging, bone degradation was analyzed in a total of three rats from each group and the radiographic images were collected and preserved for further examinations.

\section{Real-time polymerase chain reaction (RT-} PCR) and western blot analysis

Total RNA was isolated from cartilage tissues in the experimental groups and the mRNA expression levels of COX-2, Col2A, transforming growth factor beta (TGF- $\beta$ ), matrix metalloproteinases 1, 3, 13 (MMP-1, MMP-3 and MMP-13), and bone morphogenetic protein 2 (BMP-2) were determined. The sequences of primers used are shown in Table 1. Total RNA was transcribed to cDNA using a cDNA synthesis kit (Cell signaling technologies, Beijing, China). The relative mRNA expression levels were calculated using the 2- $\Delta \Delta C T$ method. Total protein was extracted from the tissues using RIPA lysis buffer solution containing phosphatase and protease inhibitors. The isolated proteins were resolved in $10 \%$ SDSPAGE, followed by electro-transfer onto polyvinylidene difluoride membranes. Then, the membranes were incubated overnight at $4{ }^{\circ} \mathrm{C}$ with primary antibodies for MMP-1, MMP-3, MMP-13 (Abcam, UK), and $\beta$-actin. Thereafter, the membranes were incubated with secondary antibody conjugated with horseradish peroxidase. The signals were observed with enhanced chemiluminescence, and the expression levels were calculated in relation to that of $\beta$-actin which served as internal control.

\section{Immuno-histochemical evaluation}

Sections of the femur and tibia of both legs were placed in $10 \%$ buffered formalin for 2 days after knee joint disarticulation. Thereafter, the bones were decalcified and preserved in paraffin wax. Pathological changes in the bone slices were determined using $H$ \& $E$ staining. In the next stage, fragments of the bone were stained with $\mathrm{H}$ \& $E$ for examination of microscopic changes in cartilage and glycosaminoglycans. The pathological changes evaluated using the Mankin scoring system [8].

\section{Cytokine inflammation assay}

Using inflammation assay (LEGENDplex rat) kits, simultaneous assays of 10 cytokines from the serum samples were performed. Out of the 10 cytokines, only IL-6, IFN-g, IL-1 $\beta$, and monocyte chemoattractant protein-1 (MCP-1) or CCL2 showed slight statistical deviations from the normal levels of the control group under various conditions. The cytokine assays were carried out in accordance with the instructions of the kit manufacturer. LEGENDplex version 8.0 software was used for analyzing the data. The levels of CTX1 and PGE2 were determined with ELISA kits as per the manufacturer's instructions.

Table 1: Primers used for RT-PCR

\begin{tabular}{|c|c|c|}
\hline \multicolumn{2}{|l|}{ Target } & Sequence (5' to $\left.3^{\prime}\right)$ \\
\hline \multirow[t]{2}{*}{ TGF- $\beta$} & Forward & GCTGCACGCCGGAGTCGGCC \\
\hline & Reverse & CCTACTAAATGGATTGCGCA \\
\hline \multirow[t]{2}{*}{ COX-2 } & Forward & CAATGGACCTTACGGACCTACTT \\
\hline & Reverse & TTGAACCGGTACCTCCAGTCAG \\
\hline \multirow[t]{2}{*}{ BMP2 } & Forward & ACCTAACCGGTACCGCCAATCA \\
\hline & Reverse & AACCTTAGCCATCGGATCGGTA \\
\hline \multirow[t]{2}{*}{$\beta$-actin } & Forward & CCTTAGTACCATCGACCAATGG \\
\hline & Reverse & GGTACCTTAACCATGCATCGACA \\
\hline \multirow[t]{2}{*}{ MMP13 } & Forward & TTACCAGGGACCTACCAGGCAA \\
\hline & Reverse & AAGGCCTAGGTTACAATCGCCA \\
\hline \multirow[t]{2}{*}{ Col2A } & Forward & GGAACCTAGGCTACATTACGAC \\
\hline & Reverse & AACCTGGGTACTTACTGAACTG \\
\hline
\end{tabular}




\section{Statistical analysis}

The results are expressed as mean \pm standard error of the mean (SEM). Statistical analyses were done with ANOVA and Student's t-test using GraphPad Prism 8.1 version (Biomatters, Ltd, USA). Values of $p<0.05$ indicated statistically significant differences.

\section{RESULTS}

Body weight, paw withdrawal threshold, and weights of spleen and uterus

At baseline, there were no significant variations in body weight amongst the groups (Figure $1 \mathrm{~A}$ ). However, ovariectomy resulted in marked weight gain in the rats, reaching $37 \%$ after 4 weeks in MIA-induced OA rats treated with vehicle $(p<$ 0.05; Figure1 A). However, no marked decreases in body weight were noticed in rats treated with luteolin (50 and $100 \mathrm{mg} / \mathrm{kg}$ ) and aceclofenac at the end of the 4-week study, when compared to MIA-induced OA and ovariectomized rats treated with vehicle (Figure $1 \mathrm{~A}$ ). In addition, no significant weight gain was observed in shamoperated control rats after 4 weeks of treatment, relative to vehicle-treated rats.

The uterus wet weight was determined at the completion of the study. Ovariectomy resulted in significant uterine degeneration in MIA-induced $\mathrm{OA}$ rats treated with vehicle, when compared to age-matched sham-operated rats (Table 2). After the 4-week study period, no significant differences in uterus weights were observed amongst the luteolin-treated (50 and $100 \mathrm{mg} / \mathrm{kg}$ ), aceclofenac-treated, and vehicle-treated groups. In contrast, rats in the sham-operated group had significantly larger uteri than the vehicle-treated group $(p<0.05)$.
There was marked elevation in the paw withdrawal threshold of the vehicle-treated MIAinduced OA rats $(p<0.05)$. However, luteolin treatment led to dose-dependent decreases in the paw withdrawal threshold of MIA-induced OA rats $(p<0.05)$. With the administration of aceclofenac, there were marked decreases in the paw withdrawal threshold of the MIA-induced $O A$ rats, when compared to the corresponding level for the vehicle-treated group (Figure $1 \mathrm{~B}$ ). At the end of the experiment, the spleen weights in the various groups were comparable. A slight increase in the spleen weight of the aceclofenactreated group was observed, relative to the control group (Figure $1 \mathrm{C}$ ).
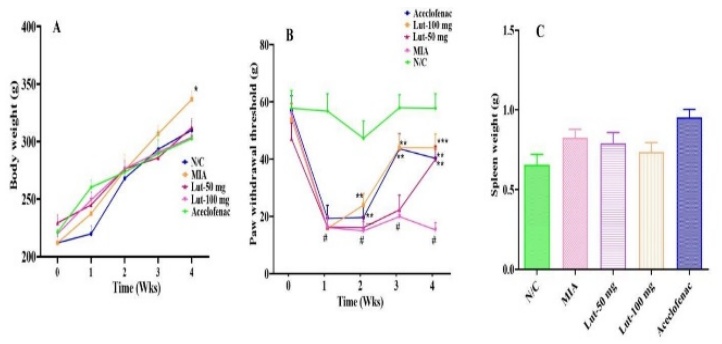

Figure 1: Body weight, paw withdrawal threshold and spleen weights of MIA-induced OA and ovariectomized rats treated with luteolin and aceclofenac for 28 days. (A) Weights of rats assessed once a week before the experiment was performed. (B) Paw withdrawal threshold of MLA-induced OA ovariectomized rats given 50 or $100 \mathrm{mg} / \mathrm{kg} / \mathrm{bwt}$ luteolin, as measured using von Frey filament testing. (C) Spleen weights of MLAinduced $\mathrm{OA}$ and ovariectomized rats given luteolin and aceclofenac, relative to control group. ${ }^{*} P<0.05$; ${ }^{* *} p<$ $0.01 ;{ }^{* * *} p<0.001$, relative to MIA-induced OA and ovariectomized rats; ${ }^{*} p<0.001$, relative to sham control rats. $(\mathrm{N} / \mathrm{C}=$ normal control; Lut-50 = luteolin dose of $50 \mathrm{mg} / \mathrm{kg} / \mathrm{bwt}$; Lut-100 = luteolin dose of 100 $\mathrm{mg} / \mathrm{kg} / \mathrm{bwt} ; \mathrm{MIA}=$ monoiodoacetate-induced $\mathrm{OA}$ in ovariectomized rats)

Table 2: Weight changes after 4 weeks of luteolin and aceclofenac treatments of MIA-induced OA in ovariectomized rats for 28 days

\begin{tabular}{llcc}
\hline Group & Treatment & $\begin{array}{c}\text { Weight }(\mathbf{g}) \text { of the } \\
\text { uterus at the end of } \\
\text { the study }\end{array}$ & P-value \\
\hline 1 & $\begin{array}{l}\text { Sham control rats } \\
\text { MIA-induced OA in ovariectomized rats } \\
\text { (vehicle-treated) }\end{array}$ & $0.76 \pm 0.05$ & - \\
2 & $\begin{array}{l}\text { MIA-induced OA in ovariectomized rats } \\
\text { treated with luteolin }(50 \mathrm{mg} / \mathrm{kg})\end{array}$ & $0.17 \pm 0.09^{* * *}$ & $<0.001$ \\
3 & $\begin{array}{l}\text { MIA-induced OA in ovariectomized rats } \\
\text { treated with luteolin (100 } \mathrm{mg} / \mathrm{kg})\end{array}$ & $0.14 \pm 0.06$ & $>0.05$ \\
4 & $\begin{array}{l}\text { MIA-induced OA in ovariectomized rats } \\
\text { treated with aceclofenac-10 } \mathrm{mg} / \mathrm{kg}\end{array}$ & $0.16 \pm 0.07$ & $>0.05$ \\
\hline 5
\end{tabular}

Values are mean \pm SEM. ${ }^{* *} P<0.001$, sham-operated control rats vs MIA-induced OA (vehicle treated) rats. No significant differences were noticed in uterus weight between vehicle-treated groups and luteolin- and aceclofenac-treated groups 

Thickness of the knee joint and X-ray
evaluation

Ovariectomized rats that received intra-articular injection of MIA in the knee joint had knee swelling that disappeared after oral administration of either aceclofenac $(50 \mathrm{mg} / \mathrm{kg})$ or luteolin $(100 \mathrm{mg} / \mathrm{kg})$. Figure 2 A shows the knee joint thickness $(\mathrm{mm})$ of rats in the various groups, as determined using a vernier caliper. Articular cartilage deterioration caused by the administration of MIA in ovariectomized rats was assessed with the aid of X-ray radiography (Figure $2 \mathrm{~B}$ ). The bone tissues were severely degraded in vehicle-treated rats, when compared to sham-operated control rats $(p<0.01)$. However, treatment of the rats with luteolin (50 and $100 \mathrm{mg} / \mathrm{kg}$ ) or aceclofenac resulted in significant suppression of degradation of articular cartilage and subchondral bone $(p<0.05)$. Therefore, luteolin treatment markedly relieved MIA caused osteoarthritis symptoms in ovariectomized rats.
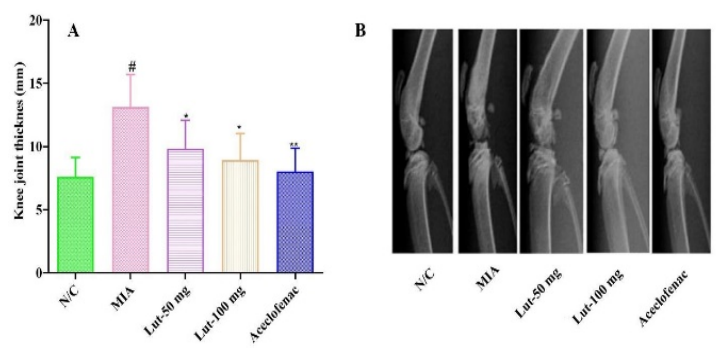

Figure 2: Effects of luteolin and aceclofenac on knee joint swelling and degradation of bone tissue in MIAinduced $O A$ and ovariectomized rats. (A) Knee joint thicknesses of rats in the various groups, as measured using a vernier calliper. (B) Statuses of femur and tibia of MIA-induced $\mathrm{OA}$ and ovariectomized rats after treatment with luteolin (50 and $100 \mathrm{mg} / \mathrm{kg}$ ) or aceclofenac $(10 \mathrm{mg} / \mathrm{kg})$, as examined using $X$-ray radiography. ${ }^{\sharp} P<0.01$, compared to normal control; ${ }^{*} p$ $<0.05$; ${ }^{* *} p<0.01$, compared to MIA-induced OA and ovariectomized rats. $(\mathrm{N} / \mathrm{C}=$ normal control; Lut $-50=$ luteolin dose of $50 \mathrm{mg} / \mathrm{kg} / \mathrm{bwt}$; Lut-100 = luteolin dose of $100 \mathrm{mg} / \mathrm{kg} / \mathrm{bwt}$; MIA = monoiodoacetate-induced OA in ovariectomized rats)

\section{Histopathological features}

Results from $\mathrm{H} \& \mathrm{E}$ staining revealed that the articular cartilage of the sham-operated control group was healthy, while there was a significant loss of chondrocytes leading to cartilage deterioration in the vehicle-treated MIA-induced OA group. In comparison, the groups treated with luteolin at doses of 50 and $100 \mathrm{mg} / \mathrm{kg}$ had improved cartilage, with significant increases in the number of chondrocytes, and marked cartilage repair. Luteolin produced a dosedependent inhibitory effect on cartilage deterioration. Similarly, aceclofenac treatment led to reductions in loss of cartilage and subchondral bone in MIA-induced OA rats.

The Mankin scoring system was used to assess the cartilage matrix, cartilage surface, and chondrocytes (Figure $3 \mathrm{~B}$ ). The levels of these parameters were significantly greater in the vehicle-treated rats than in the sham rats, and cartilage erosion was three-fold higher in the vehicle-treated rats. Compared to shamoperated rats, ovariectomy had a significantly larger effect on cartilage degradation in the rats, particularly in the medial and lateral femurs $(p=$ $0.001)$. In the vehicle-treated group, the total cartilage erosion demonstrated markedly more serious surface erosion than in the shamoperated group $(p=0.001)$. However, treatment with luteolin or aceclofenac produced reduction in cartilage erosion, when compared to the vehicle-treated group. The use of luteolin and aceclofenac resulted in significantly reduced surface erosion characteristics in the medial tibia, lateral and medial femurs, and in knee joints, when compared to the vehicle-treated group. In addition, increased levels of proteoglycans were seen in the cartilage and bone tissue in the luteolin-treated groups, relative to the vehicle-treated group (Figure $3 \mathrm{~A}$ ).

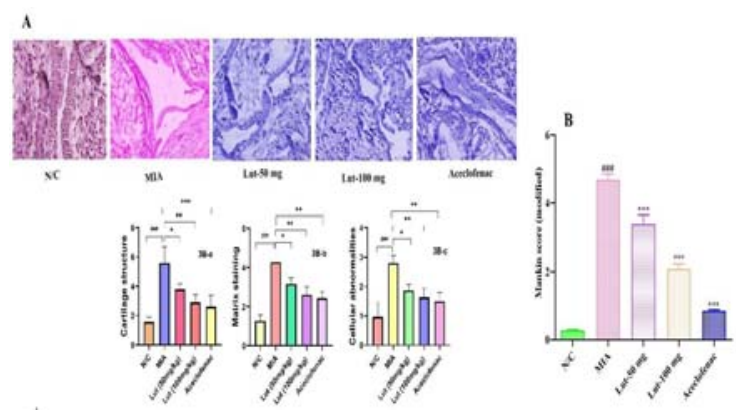

Figure 3: Histological evaluation of protective effect of luteolin against MIA-induced OA in ovariectomized rats after treatment for 28 days. (A) Mitigation of pathological lesions of MIA-induced OA in ovariectomized rats treated with luteolin doses of 50 and $100 \mathrm{mg} / \mathrm{kg}$, and aceclofenac $(10 \mathrm{mg} / \mathrm{kg})$ for 4 weeks. (B) Bar graph of lesions in joints, as evaluated using the modified Mankin's scoring system, indicating combined scores for cartilage structure (3B-a), matrix staining (3B-b) and cellular abnormalities (3B-c) of different groups via assessment of cartilage matrix, cartilage surface and chondrocytes, respectively. ${ }^{\# \#} P$ $<0.01$, compared to normal control; ${ }^{*} p<0.05$, ${ }^{* *} p<$ $0.01,{ }^{* * *} p<0.001$, compared with vehicle-treated MIAinduced $\mathrm{OA}$ and ovariectomized rats. $(\mathrm{N} / \mathrm{C}=$ normal control; Lut-50 = luteolin dose of $50 \mathrm{mg} / \mathrm{kg} / \mathrm{bwt}$; Lut$100=$ luteolin dose of $100 \mathrm{mg} / \mathrm{kg} / \mathrm{bwt} ; \mathrm{MIA}=$ monoiodoacetate-induced $\mathrm{OA}$ in ovariectomized rats). Scale bar $=400 \mu \mathrm{m}$

Trop J Pharm Res, October 2021; 20(10): 2103 
Effect of luteolin on mRNA expressions of MMP-1, MMP-3, MMP-13, Cox 2, and TGF- $\beta$ in chondrocytes

The anti-inflammatory and anti-catabolic effects of luteolin on chondrocyte mRNA expressions were investigated with RT-PCR. The protein expression levels of anti-inflammatory mediators (Cox 2 and TGF-) and catabolic variables (MMP1, MMP-3 and MMP-13) were determined. Results from Western blot assay (Figure 4) indicated that MIA-induced OA in ovariectomized rats significantly increased the protein levels of COX 2, TGF- $\beta$, MMP-1, MMP-3, and MMP-13, relative to the sham-treated group $(p<0.05)$. However, luteolin (50 and $100 \mathrm{mg} / \mathrm{kg}$ ) or aceclofenac treatment significantly decreased the protein expressions of COX 2, TGF- $\beta$, MMP$1, \mathrm{MMP}-3$, and MMP-13 in rat chondrocytes. The BMP-2 expression was significantly increased after treatment with luteolin or aceclofenac $(p<$ $0.01)$.

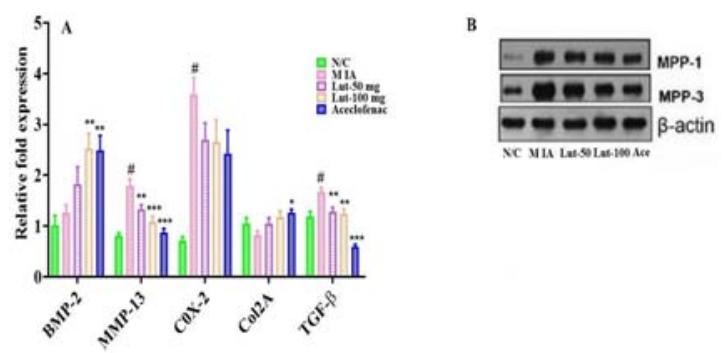

Figure 4: Effects of luteolin and aceclofenac on the mRNA expressions of different bone growth markers in chondrocytes. (A) Bar chart showing relative expressions of different bone growth markers. (B) Effects of luteolin and aceclofenac on relative protein expressions of MPP-1 and MPP-3. ${ }^{\#} P<0.01$, relative to normal control; ${ }^{*} p<0.05,{ }^{* *} p<0.01,{ }^{* *} p<0.001$, compared to MIA-induced OA. (N/C = normal control; Lut-50 = luteolin dose of $50 \mathrm{mg} / \mathrm{kg} / \mathrm{bwt}$; Lut-100 = luteolin dose of $100 \mathrm{mg} / \mathrm{kg} / \mathrm{bwt} ; \quad \mathrm{MIA}=$ monoiodoacetate-induced $\mathrm{OA}$ in ovariectomized rats)

\section{Effects of luteolin and aceclofenac on inflammatory mediators}

The effect of ovariectomy on bone formation was reflected in increases in serum CTX-I concentrations, as shown in Figure 5 and Figure 6. The levels of CTX-I were significantly higher in the vehicle-treated rats than in the shamoperated rats (Figure 5). However, luteolin at doses of 50 and $100 \mathrm{mg} / \mathrm{kg}$, and aceclofenac suppressed bone resorption, as indicated in significant reductions in levels of the bone resorption marker, CTX-I $(p<0.05$; Figure 5).
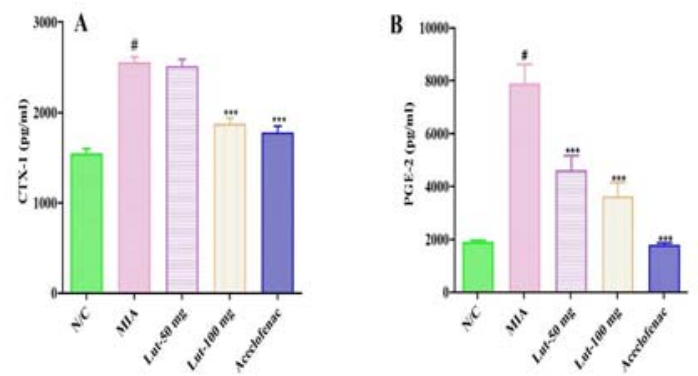

Figure 5: Effects of luteolin and aceclofenac on the serum levels of inflammatory mediators. (A) CTX1 levels, and (B) PGE2 levels, as measured using ELISA. ${ }^{\#} P<0.01$, compared to normal control; ${ }^{* * *} p<$ 0.001, compared to MIA-induced OA. (N/C = normal control; Lut-50 = luteolin dose of $50 \mathrm{mg} / \mathrm{kg} / \mathrm{bwt}$; Lut$100=$ luteolin dose of $100 \mathrm{mg} / \mathrm{kg} / \mathrm{bwt} ; \mathrm{MIA}=$ monoiodoacetate-induced $\mathrm{OA}$ in ovariectomized rats)

Figure 5 shows that there were higher PGE2 levels in vehicle-treated (MIA-induced $O A$ ) rats than in sham-operated control rats, indicating that fever and inflammation were linked with $O A$ induction, and may be reduced with luteolin and aceclofenac. There were up-regulated expressions of MCP-1/CCl-2 and IL- 6 , as well as down-regulation of IFN-y expression. However, treatment with aceclofenac or luteolin (50 and $100 \mathrm{mg} / \mathrm{kg}$ ) regulated and stabilized inflammatory cytokine levels in a dosedependent manner (Figure 6).
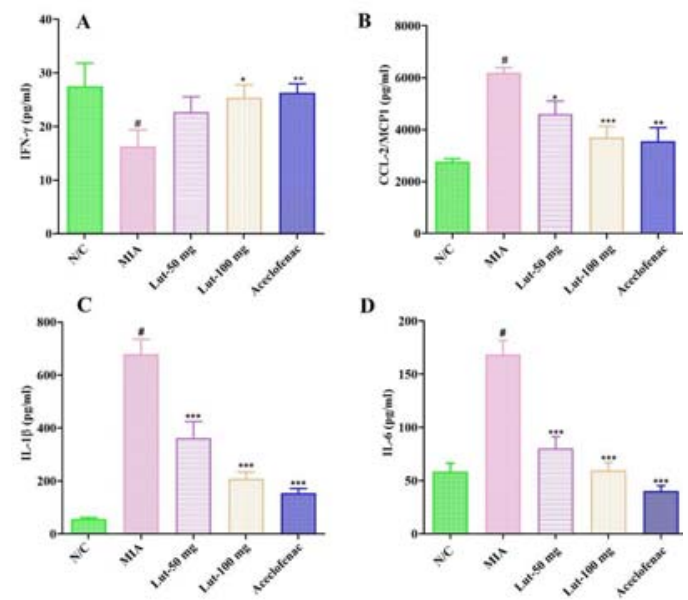

Figure 6: Effects of luteolin and aceclofenac on levels of inflammatory cytokines. Only four inflammatory cytokines differed significantly between shamoperated control group and vehicle-treated (MIAinduced OA) group. (A) IFN-Y (B) CCL-2/MCP1 (C) IL$1 \beta$ and (D) IL-6. ${ }^{\#} P<0.001$, compared to normal control; ${ }^{*} p<0.05,{ }^{* *} p<0.01,{ }^{* *} p<0.001$, compared to MIA-induced OA. (N/C = normal control; Lut-50 = luteolin dose of $50 \mathrm{mg} / \mathrm{kg} / \mathrm{bwt}$; Lut-100 = luteolin dose of $100 \mathrm{mg} / \mathrm{kg} / \mathrm{bwt} ; \mathrm{MIA}=$ monoiodoacetate-induced $\mathrm{OA}$ in ovariectomized rats) 


\section{DISCUSSION}

The current study investigated the morphology of the cartilage and its turnover in shamoperated and MIA-triggered OA in ovariectomized rats, to find out whether a mild dose of intra-articularly-administered MIA and inhibition of production of endogenous estrogen by ovariectomy affected articular cartilage integrity and turnover. When MIA is injected intra-articularly, it inhibits glyceraldehyde-3phosphate dehydrogenase, thereby disrupting glycolysis and enhancing chondrocyte mortality. The results obtained in this study indicated that MIA-induced OA caused a significant rise in articular cartilage erosion in ovariectomized rats. A previous study established the pharmacological significance of luteolin, but it did not report on its impact on MIA-induced OA in ovariectomized rat model [9]. This study was undertaken to understand the pharmacological effects of luteolin on OA induced by MIA in ovariectomized rats. It has demonstrated that the administration of exogenous luteolin or aceclofenac in MIA-induced OA and ovariectomized rats suppressed articular cartilage erosion and restored its integrity.

At the end of the study after 4 weeks of luteolin (50 and $100 \mathrm{mg}$ ) and aceclofenac treatments, the sham control group showed a significant increase in uteri weights, thereby demonstrating the uterotropic effects of ovay-released estrogen, since the ovaries were exposed but not removed. However, no significant differences were seen either in body and uterus weight gains after treatment with luteolin or aceclofenac, when compared to the vehicletreated group. In addition, no major differences in spleen weights in untreated (sham-operated and vehicle-treated groups) and treated groups were observed. This demonstrates that luteolin produced no side effects or adverse effects.

In addition to subchondral bone degradation in ovariectomized rats, the knee joint was severely swollen, and synovium was observed after one week of intra-articular injection of MIA [10]. Pain is one of the major causes of OA, which is why the capacity to bear weight while walking is usually examined. When compared to the vehicle-treated group, luteolin treatment resulted in a decrease in paw withdrawal threshold, thereby demonstrating the anti-inflammatory properties of luteolin.

The protective effect of luteolin was determined using H\&E staining. The changes observed in ovariectomized rats (vehicle-treated group) with MIA-induced OA were most pronounced in cartilage erosion, followed by proteoglycan loss. There were severe erosive alterations such as fibrillation and enhanced vascularization, which could be due to the combined effects of MIA and ovariectomy. When compared to other OA models, the histology of knee articular cartilage in the MIA-induced OA in rats was significantly different in terms of meniscal tear and ligament transection [11]. Morphological and ultrastructural analysis revealed that luteolin significantly decreased cartilage matrix, cartilage surface, and chondrocytes, resulting in a lower Mankin's histological score. Moreover, the percentage of staining was proportional to the mitigating effect of luteolin against MIA-induced OA chondrocytes, implying that luteolin was effective in protecting chondrocytes. Thus, the MIA-triggered osteoarthritis in ovariectomized rats may be an ideal model for studying OA.

In OA-induced rats, serum CTX-1 levels were markedly raised, when compared to normal control rats, suggesting bone resorption. These results are consistent with the anticipated increase in bone resorption caused by ovariectomy [12]. These findings are in agreement with findings in other studies which showed that ovariectomy, which is similar to menopause transition, promotes arthritis [3]. In addition, the results indicate that ovariectomy in rats results in oestrogen deficiency which elicits skeletal metabolic changes associated with menopause. These findings corroborate those of other studies [12]. The increase in weight and reduction in uterus mass observed following ovariectomy are consistent systemic effects of oestrogen withdrawal. However, the study results showed that the that the mineral content of the bone can be restored by administering luteolin to osteoporotic ovariectomized rats [13].

The link between aetiology of $O A$ and inflammation has been revealed in several previous investigations [14]. The major and one of the most essential components of COX activity is PGE2 which acts as an inflammatory mediator and exerts an inhibitory effect on a variety of inflammatory disorders. In addition, PGE2 upregulates MMPs and a few inflammatory cytokines. The effect of luteolin on serum PGE2 levels was studied in the treatment groups. The results showed that, as expected, PGE2 levels were significantly increased in MIA-triggered OA in ovariectomized rats. However, this effect was significantly decreased following luteolin administration.

Matrix metalloproteinases (MMPs) are a family of proteolytic enzymes involved in the regulation of OA. They promote the degradation of articular 
cartilage components such as collagens I and II, which is a critical step in controlling articular cartilage formulation and ECM degradation. The balance between the synthesis and degradation of ECM is disrupted when MMPs are released uncontrollably. The aetiology of $\mathrm{OA}$ is due to increased protein expressions of the MMP-1, MMP-2, and MMP-9. Recent evidence indicates that debilitating menisci secrete significantly high levels of matrix-degrading enzymes such as MMP-1, MMP-3, and MMP-8, which are thought to promote OA [15]. In particular, MMP-13 is thought to play a significant role in the development of $\mathrm{OA}$ due to its cleavage of collagen-II. In the present study, there were increases in protein expression levels of MMP-1, MMP-3, and MMP-13, and collagen I was degraded in rat chondrocytes. However, luteolin inhibited MMP up-regulation and collagen I degradation. The mRNA expression levels of MMP-1, MMP-3 and MMP-13 were decreased significantly and dose-dependently by luteolin treatment. These results show that the antidegradative effect of luteolin could be attributable to the inhibition of MMPs. This study has demonstrated the luteolin exerted antiinflammatory effect by suppressing the mRNA expressions of inflammatory mediators that affect knee joint chondrocytes.

A previous study found that in the early stages of $\mathrm{OA}$, cartilage degradation is controlled by IFN-Y through regulation of the expression of $\mathrm{IL}-1 \beta$ and reduction of the production of MMPs [16]. Interestingly, this study showed that luteolin treatment increased the levels of the inflammatory cytokines IL- 6 and IL- $1 \beta$, when compared to previous studies. Moreover, IFN-y levels were elevated and cartilage chondrocyte apoptosis was reduced after treatment with luteolin.

There are certain limitations in this study due to budget and time constraints. Firstly, the researchers were unable to conduct additional in vitro and in vivo studies linking luteolin to other inflammatory mediators such as IL-1, IL-6, and TNF- $\alpha$. Secondly, there was no comparisons between luteolin and any other flavonoid such as quercetin or ferulic acid. It is critical to further understand the genetic effects of luteolin through studies comparing it with other flavonoids. Thirdly, in vitro studies using chondrocytes were not conducted to corroborate the findings from the in vivo research.

\section{CONCLUSION}

This study specifically shows that $O A$ is effectively regulated by decreasing the levels of inflammatory cytokines or chemokines in rats. Luteolin protects against cartilage deterioration by suppressing the mRNA expression levels of MMP-1, MMP-3, MMP-13, and COX-2, and by inhibiting the pro-inflammatory cytokines IL-6, PGE2, IL- $\beta$ and IFN $\gamma$ which generally function as anti-inflammatory agents. These findings indicate that luteolin exerts anti-arthritic effect on MIAmediated $\mathrm{OA}$ in ovariectomized rats, and may also play role in the management of $O A$.

\section{DECLARATIONS}

\section{Conflict of Interest}

No conflict of interest associated with this work.

\section{Contribution of Authors}

The authors declare that this work was done by the authors named in this article and all liabilities pertaining to claims relating to the content of this article will be borne by them.

\section{Open Access}

This is an Open Access article that uses a funding model which does not charge readers or their institutions for access and distributed under the terms of the Creative Commons Attribution License (http://creativecommons.org/licenses/by/ 4.0) and the Budapest Open Access Initiative (http://www.budapestopenaccessinitiative.org/rea d), which permit unrestricted use, distribution, and reproduction in any medium, provided the original work is properly credited.

\section{REFERENCES}

1. Wojdasiewicz $P$, Poniatowski $Ł A$, Szukiewicz $D$. The role of inflammatory and anti-inflammatory cytokines in the pathogenesis of osteoarthritis. Mediat Inflam 2014:2014.

2. Charlier E, Relic B, Deroyer C, Malaise O, Neuville S, Collée J, Malaise MG, De Seny D. Insights on molecular mechanisms of chondrocytes death in osteo-arthritis. Int J Mol Sci 2016; 17:2146.

3. VanDelden JJ, Vander G, Raaf R. Revised CIOMS International Ethical Guidelines for Health-Related Research Involving Humans. JAMA 2017; 317(2): 135136

4. Bendele A. Animal models of osteoarthritis. J Musculoskelet Neuronal Interact 2001; 1:363e76.

5. Høegh-Andersen P, Tankó LB, Andersen TL, Lundberg CV, Mo JA, Heegaard A-M, et al. Ovariectomized rats as a model of postmenopausal osteoarthritis: validation and application. Arthritis Res Ther 2004; 6:R169.

6. SF Nabavi, N Braidy, O Gortzi, E Sobarzo-Sanchez, M Daglia, K SkalickaWozniak, SM Nabavi. Luteolin as an 
anti-inflammatory and neuroprotective agent: a brief review, Brain Res. Bull. 119 (2015) 1-11.

7. J Xiong, $K$ Wang, C Yuan, $R$ Xing, J Ni, G Hu, F Chen, $X$ Wang. Luteolin protects mice from severe acute pancreatitis by exerting HO-1-mediated antiinflammatory and antioxidant effects, Int. J. Mol. Med. 39 (2017) 113-125.

8. N Xia, G Chen, M Liu, X Ye, Y Pan, J Ge, Y Mao, H Wang, J Wang, $S$ Xie. Anti-inflammatory effects of luteolin on experimental autoimmune thyroiditis in mice, Exp. Ther. Med. 12 (2016) 4049-4054.

9. Park JG, Yi Y-S, Hong YH, Yoo S, Han SY, Kim E, et al. Tabetri (Tabebuia avellanedae ethanol extract) ameliorates osteoarthritis symptoms induced by monoiodoacetate through its Anti-Inflammatory and Chondroprotective Activities. Mediat Inflamm 2017.

10. Kim JH, Yi Y-S, Kim M-Y, Cho JY. Role of ginsenosides, the main active com-ponents of Panax ginseng, in inflammatory responses and diseases. J Ginseng Res 2017; 41:435e43.

11. Kim Y, Kim E-h, Lee KS, Lee K, Park SH, Na SH, Ko C, Kim J, Yooon YW. The effects of intra-articular resiniferatoxin on monosodium iodoacetate-induced osteoarthritic pain in rats. Korean J Physiol Pharmacol 2016; 20:129e36.

12. Gong Y-S, Chen J, Zhang Q-Z, Zhang J-T. Effect of 17boestradiol and ginsenoside on osteoporosis in ovariectomised rats. J Asian Nat Prod Res 2006; 8: $649 e 56$.

13. Janusz MJ, Bendele AM, Brown KK, Taiwo YO, Hsieh L, Heitmeyer SA: Induction of osteoarthritis in the rat by surgical tear of the meniscus: Inhibition of joint damage by a matrix metalloproteinase inhibitor. Osteoarthritis Cartilage 2002, 10:785-791

14. Marchev AS, Dimitrova PA, Burns AJ, Kostov RV, Dinkova-Kostova AT, Georgiev MI. Oxidative stress and chronic inflammation in osteoarthritis: can NRF2 counteract these partners in crime? Ann N Y Acad Sci 2017; 1401:114e 35.

15. GQ Zeng, $A B$ Chen, $W$ Li, JH Song, CY GAO. High MMP-1, MMP-2, and MMP-9 protein levels in osteoarthritis, Genet. Mol. Res. 2015; 14: 14811-14822.

16. H Li, D Wang, Y Yuan, J Min. New insights on the MMP13 regulatory network in the pathogenesis of early osteoarthritis, Arthritis Res. Ther. 2017; 19: 248 